\title{
A change in recording tooth notation
}

The BDJ has traditionally adopted the Palmer tooth notation as the first choice for recording individual teeth in papers and articles. This system, very familiar to dentists in the UK, is reproduced below for both adult and deciduous teeth.

$$
\begin{aligned}
& \begin{array}{l|ll|l}
87654321 & 12345678 & E D C B A & A B C D E \\
\hline 87654321 & 12345678 & \text { EDCBA } & A B C D E
\end{array}
\end{aligned}
$$

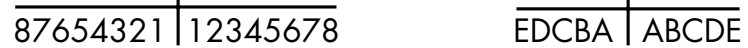

In future we are changing the actual way the Palmer system is written because of difficulties of converting the familiar grid format to our website. Instead the position on the grid will be written using the shorthand UR for upper right, UL for upper left, LL for lower left and LR for lower right.

Thus 7 becomes UR7 and $\longdiv { 5 }$ is written as LL5. Groups of teeth will be recorded as best we can, so for example 54 will become UR5 and UR4, while 2345 will be written as UL2 to UL5.

Obviously the same will apply to deciduous teeth, for example E will be written as URE.

The FDI notation will still be written in brackets after the Palmer notation, using the familiar FDI notation as described below:

$$
\begin{array}{ll|l|l|l|l|l}
1817161514131211 & 2122232425262728 & \text { for adult teeth }
\end{array}
$$

\begin{tabular}{l|l|l}
\hline 4847464544434241 & 3132333435363738
\end{tabular}

and $\quad$\begin{tabular}{r|ll}
5554535251 & 6162636465 \\
\hline 8584838281 & 7172737475
\end{tabular}$\quad$ for deciduous teeth

Thus using both systems, 7$]$ will be written as UR7 (17) and $\sqrt{5}$ will become LL5 (35). 